\title{
Proton emission half-lives within a Gamow-like model
}

\author{
A. Zdeb ${ }^{1, a}$, M. Warda ${ }^{1}$, C.M. Petrache ${ }^{2}$, and K. Pomorski ${ }^{1}$ \\ 1 Katedra Fizyki Teoretycznej, Uniwersytet Marii Curie-Skłodowskiej, ul. Radziszewskiego 10, 20-031 Lublin, Poland \\ 2 Centre de Sciences Nucléaires et Sciences de la Matière, Université Paris-Sud and CNRS/IN2P3, Université Paris-Saclay \\ 91405 Orsay, France
}

\author{
Received: 14 July 2016 / Revised: 7 September 2016 \\ Published online: 26 October 2016 \\ (c) The Author(s) 2016. This article is published with open access at Springerlink.com \\ Communicated by F. Nunes
}

\begin{abstract}
Proton emission is described using a model which has previously given good results in the description of $\alpha$ and cluster radioactivity. The simple phenomenological formalism, based on the Gamow theory for alpha decay, is now extended by including the centrifugal term. The model contains only one parameter: the effective nuclear radius constant. Its value was once found for alpha and cluster emitters. A good agreement with the experimental half-lives for proton radioactivity is achieved without any additional fitting procedures to the data for proton emission.
\end{abstract}

\section{Introduction}

The decay of ${ }^{53} \mathrm{Co}$ from its isomeric state was the first experimental observation of proton radioactivity $[1,2]$. The proton emission from the ground state of mother nuclei with masses in the range $110 \leq A \leq 150$ was measured in the early 1980s [3]. This decay mode is typical for the odd- $Z$ emitters beyond the proton drip line. The associated half-lives range from $10^{-6} \mathrm{~s}$ to a few seconds. The investigation of this process provides information on the structure of very neutron-deficient nuclei which are far from the $\beta$-stability region (shell structure, coupling between bound and unbound nuclear states). It also allows one to obtain spectroscopic properties of the nucleus due to the unpaired proton.

Up to now several attempts have been made to describe this phenomenon theoretically, which have been discussed in review papers [4-6]. The distorted wave Born approximation (DWBA) [7] and the two-potential approach (TPA) [8] represent rather sophisticated methods which allow the decay width to be found by analyzing the correlations between initial and final state wave functions in the interaction potential. The correlations between proton emission half-lives and the deformation of the mother nucleus were widely discussed in refs. [9-11]. However, these sophisticated methods often include phenomenological input, fitted parameters [4] or rough approximations $[9,10]$.

Another phenomenological attempt with the ViolaSeaborg-type formula for alpha decay and cluster radioactivity $[12,13]$ was used to describe proton emission [14-17]. This is one of the most popular formalisms due to its sim-

\footnotetext{
a e-mail: azdeb@kft.umcs.lublin.pl
}

plicity. It is a quasi-classical method based on the WKB approximation where the penetrability of the barrier is studied [18].

Recently, a simplified version of the Gamow theory was successfully applied to determine half-lives for alpha and cluster emission in ref. [19]. This method has much deeper physical basements and conserve the simplicity of the Viola-Seaborg approach. Now, we would like to show the model potentialities to describe the various decays modes even though the inclusion of the omitted microscopic effects can give more precise values. Thus we applied the same model to describe proton emission from spherical nuclei by keeping a fixed value of a single adjustable parameter. The main aim of this paper is to show that using a very simple, uniform formalism, one can evaluate half-lives for the three different decay modes: alpha, cluster and proton radioactivity. The obtained agreement with experimental data clearly shows that this model describes properly the physics of various decays (although omitted microscopic effects can give more precise values). The model can also be used for rapid evaluations of the order of magnitude of the lifetimes for different decay modes using only the $Q$-values and angular momentum $l$ of the emitting state. It is a useful tool to globally guide a survey of the known experimental masses and conceive future measurements.

\section{Model}

Analogous to alpha and cluster radioactivity, proton emission might be understood in terms of the quantummechanical tunneling of the emitted particle through 


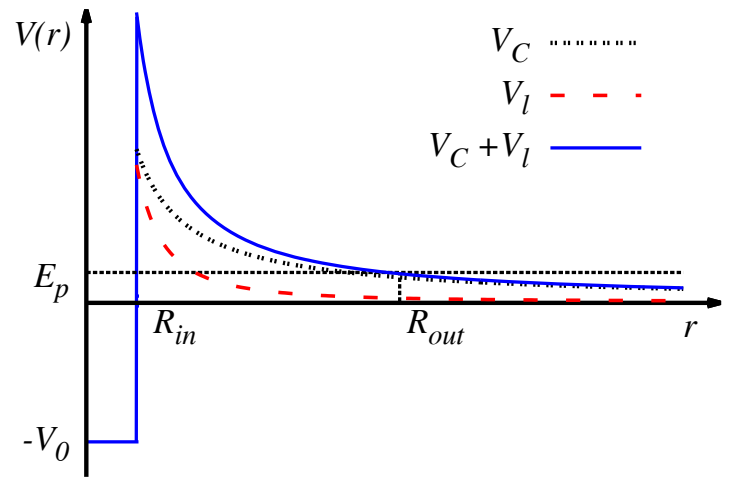

Fig. 1. Schematic plot of the potential energy as a function of the distance between the nucleus and emitted proton. The dotted lines correspond to the Coulomb $\left(V_{C}\right)$ and centrifugal $\left(V_{l}\right)$ terms.

a one-dimensional barrier. The penetration probability calculated using the WKB approximation is given by

$$
P=\exp \left[-\frac{2}{\hbar} \int_{R_{\text {in }}}^{R_{\text {out }}} \sqrt{2 \mu\left(V(r)-E_{\mathrm{p}}\right)} \mathrm{d} r\right],
$$

where $\mu=\left(m_{p} m_{A-1}\right) /\left(m_{p}+m_{A-1}\right) \simeq 938.3(A-$ 1) $/ A \mathrm{MeV} / c^{2}$ is the reduced mass of the emitted proton from the mother nucleus of mass number $A$ and $E_{p}$ is the kinetic energy of the proton, extracted from the experimental $Q$-value. $R_{i n}$ is the classical inner turning point, equal to the radius of the spherical square well in which the proton is trapped before emission, that is

$$
R_{i n}=r_{0}\left(A_{1}^{1 / 3}+A_{2}^{1 / 3}\right)
$$

where $A_{1}=1$ and $A_{2}=A-1$ for proton emission. $R_{\text {out }}$ is the exit point from the potential barrier which is determined by the condition $V\left(R_{\text {out }}\right)=E_{p}$, taking quite huge values from $60 \mathrm{fm}$ up to $110 \mathrm{fm}$.

In the description of proton emission the centrifugal barrier plays a more important role than in alpha decay, mostly because the proton carries a non-vanishing angular momentum. The impact of the centrifugal term on the potential barrier cannot be neglected as it reduces the tunneling probability and increases the half-life. Thus the proton-nucleus potential should consist of a Coulomb $V_{C}$ and a centrifugal $V_{l}$ part

$$
\begin{aligned}
V_{C}(r) & =\frac{Z_{p} Z_{d} e^{2}}{r}, \\
V_{l}(r) & =\frac{\hbar^{2} l(l+1)}{2 \mu r^{2}},
\end{aligned}
$$

where $l$ is the angular momentum carried by the valence proton which should be taken from experiment. The potential energy $V(r)$ shown in fig. 1 is given by

$$
V(r)= \begin{cases}-V_{0} & 0 \leq r \leq R_{i n}, \\ V_{C}(r)+V_{l}(r) & r>R_{i n} .\end{cases}
$$

Note that close to $R_{\text {out }}, V_{l}$ is negligible in comparison with $V_{C}$.
The proton emission half-life formula takes the following simple form:

$$
T_{1 / 2}^{p}=\frac{\ln 2}{\nu P},
$$

where $\nu$ is the frequency of assaults of the proton against the potential energy barrier. In a first approximation one may assume that it is given by the harmonic oscillator frequency present in the Nilsson potential [20]

$$
h \nu=\hbar \omega \simeq \frac{41}{A^{1 / 3}} \mathrm{MeV}
$$

The only parameter of the model is the effective nuclear radius constant $r_{0}$. It is closely related to the liquid drop model parameter [21,22], but it cannot be used to determine the size of a nucleus. It accumulates all the secondary effects not included in the simplified picture (e.g.: surface diffuseness, shape deformation, nuclear interaction, preformation factor in case of alpha and cluster emission).

\section{Results}

The comparison between the logarithms of experimental and calculated half-lives obtained using the presented model for proton emission is shown in fig. 2 and table 1 . In this work we considered 27 proton emitters (both ground and excited state emitters) from ${ }^{113} \mathrm{Cs}$ to ${ }^{177} \mathrm{Tl}$, for which all necessary input data are available. We have excluded from the analysis some isotopes used in other elaborations for which $T_{1 / 2}, Q$-value, branching ratio or $J^{\pi}$ are available but measured without reasonable accuracy. The best accuracy in proton emission half-lives data reproduction is obtaineed for nuclear radius constant $r_{0}=1.20 \mathrm{fm}$ (see fig. 3). This value is very close to the one adjusted to alpha and cluster radioactivity $\left(r_{0}=1.21 \mathrm{fm}[19]\right)$. We found that the difference between the root-mean-square deviation for $r_{0}=1.20$ and $1.21 \mathrm{fm}$ is negligible. Thus we

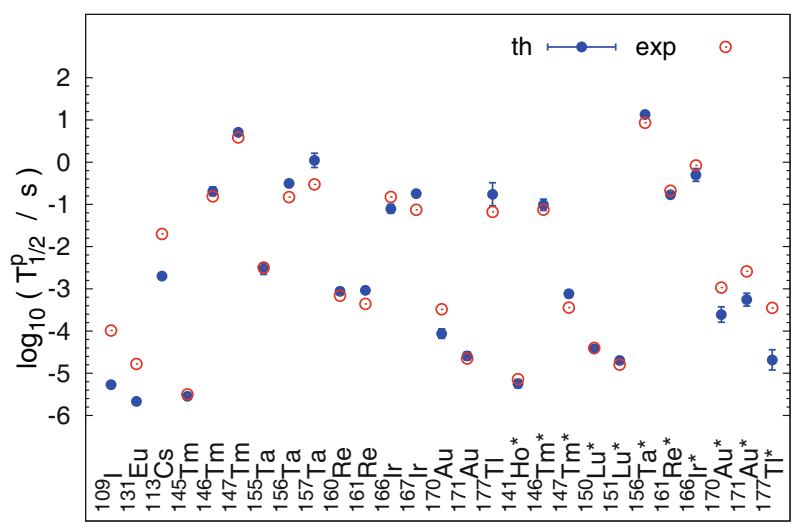

Fig. 2. The comparison between the logarithms of proton emission half-lives calculated using formula (6) with $r_{0}=$ $1.21 \mathrm{fm}$ and the experimental data [23]. The r.m.s. of theoretical data is 0.46 . 
Table 1. The comparison between the logarithms of the calculated $\left(r_{0}=1.21 \mathrm{fm}\right)$ and the experimental half-lives for proton emission. The $Q$-value and the experimental half-lives are taken from ref. [23]. The $\log _{10}\left(T_{1 / 2}^{\mathrm{cal}} / \mathrm{s}\right)$ values given in brackets refer to the results obtained after the replacement of $l_{\exp }$ by the value found in our analysis. The emission from the excited state is marked by a star.

\begin{tabular}{|c|c|c|c|c|}
\hline Nucleus & $l$ & $Q[\mathrm{keV}]$ & $\log _{10}\left(\frac{T_{1 / 2}^{\exp }}{\mathrm{s}}\right)$ & $\log _{10}\left(\frac{T_{1 / 2}^{\mathrm{cal}}}{\mathrm{s}}\right)$ \\
\hline${ }^{109} \mathrm{I}$ & $0[23]$ & $819.5(1.9)$ & -3.99 & $-5.27(-4.25)$ \\
\hline${ }^{113} \mathrm{Cs}$ & $2[23]$ & $973.5(2.6)$ & -4.78 & -5.67 \\
\hline${ }^{131} \mathrm{Eu}$ & $2[23]$ & $947.0(5.0)$ & -1.70 & $-2.70(-1.70)$ \\
\hline${ }^{145} \mathrm{Tm}$ & $5[23]$ & $1736.0(7.0)$ & -5.50 & -5.55 \\
\hline${ }^{146} \mathrm{Tm}$ & $0[24]$ & $896.0(6.0)$ & -0.81 & -0.69 \\
\hline${ }^{147} \mathrm{Tm}$ & $5[23]$ & $1059.0(3.0)$ & 0.59 & 0.71 \\
\hline${ }^{155} \mathrm{Ta}$ & $5[23]$ & $1453.0(15.0)$ & -2.49 & -2.53 \\
\hline${ }^{156} \mathrm{Ta}$ & $2[25]$ & $1020.0(5.0)$ & -0.83 & -0.50 \\
\hline${ }^{157} \mathrm{Ta}$ & $0[23]$ & $935.0(10.0)$ & -0.53 & 0.04 \\
\hline${ }^{160} \mathrm{Re}$ & $2[26]$ & $1267.0(7.0)$ & -3.16 & -3.06 \\
\hline${ }^{161} \mathrm{Re}$ & $0[23]$ & $1197.0(5.0)$ & -3.36 & -3.03 \\
\hline${ }^{166} \mathrm{Ir}$ & $2[27]$ & $1152.0(8.0)$ & -0.82 & -1.10 \\
\hline${ }^{167} \mathrm{Ir}$ & $0[27]$ & $1070.0(4.0)$ & -1.13 & -0.75 \\
\hline${ }^{170} \mathrm{Au}$ & $2[28]$ & $1472.0(12.0)$ & -3.49 & -4.06 \\
\hline${ }^{171} \mathrm{Au}$ & $0[27]$ & $1448.0(10.0)$ & -4.65 & -4.59 \\
\hline${ }^{177} \mathrm{Tl}$ & $0[23]$ & $1160.0(20.0)$ & -1.18 & -0.76 \\
\hline${ }^{141} \mathrm{Ho}^{*}$ & $0[23]$ & $1183.0(9.0)$ & -5.14 & -5.25 \\
\hline${ }^{146} \mathrm{Tm}^{*}$ & $5[24]$ & $1200.0(12.0)$ & -1.12 & -1.01 \\
\hline${ }^{147} \mathrm{Tm}^{*}$ & $2[23]$ & $1121.0(7.0)$ & -3.44 & -3.12 \\
\hline${ }^{150} \mathrm{Lu}^{*}$ & $2[29]$ & $1291.6(7.3)$ & -4.40 & -4.42 \\
\hline${ }^{151} \mathrm{Lu}^{*}$ & $2[23]$ & $1318.1(7.0)$ & -4.80 & -4.70 \\
\hline${ }^{156} \mathrm{Ta}^{*}$ & $5[26]$ & $1114.0(4.0)$ & 0.93 & 1.13 \\
\hline${ }^{161} \mathrm{Re}^{*}$ & $5[23]$ & $1320.7(6.3)$ & -0.68 & -0.77 \\
\hline${ }^{166} \mathrm{Ir}^{*}$ & $5[27]$ & $1324.0(14.0)$ & -0.08 & -0.30 \\
\hline${ }^{170} \mathrm{Au}^{*}$ & $5[28]$ & $1752.0(25.0)$ & -2.97 & -3.61 \\
\hline${ }^{171} \mathrm{Au}^{*}$ & $5[27]$ & $1703.0(20.0)$ & -2.59 & -3.26 \\
\hline${ }^{177} \mathrm{Tl}^{*}$ & $5[23]$ & $1967.0(38.0)$ & -3.45 & $-4.69(-3.39)$ \\
\hline
\end{tabular}

decided to perform calculations for proton emission halflives using the value of $r_{0}=1.21 \mathrm{fm}$. It allows to describe three different decay modes using the same value of one parameter, which makes our model more uniform without any loss of its accuracy. The isotopes ${ }^{109} \mathrm{I},{ }^{131} \mathrm{Eu}$ and ${ }^{177} \mathrm{Tl}^{*}$ were excluded from this analysis as the calculated half-lives differ by about one order of magnitude from the experimental ones. These three nuclei will be discussed below. The theoretical errors, which may arise from the experimental uncertainties of $Q$-value measurements, are also plotted in fig. 2. In most cases the error bars do not exceed the size of the points. The total r.m.s. deviation obtained for 27 proton emitters is 0.522 orders of magnitude and goes down to 0.365 when the mentioned three isotopes

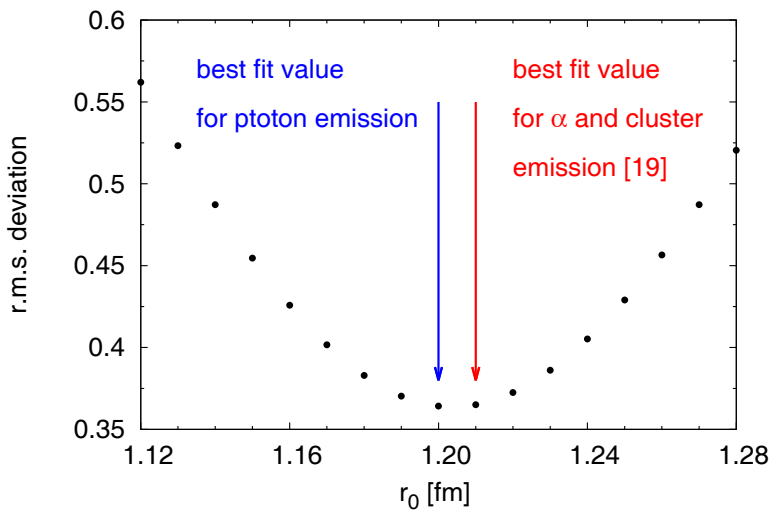

Fig. 3. The dependence of the r.m.s. deviation on the value of the $r_{0}$ parameter.

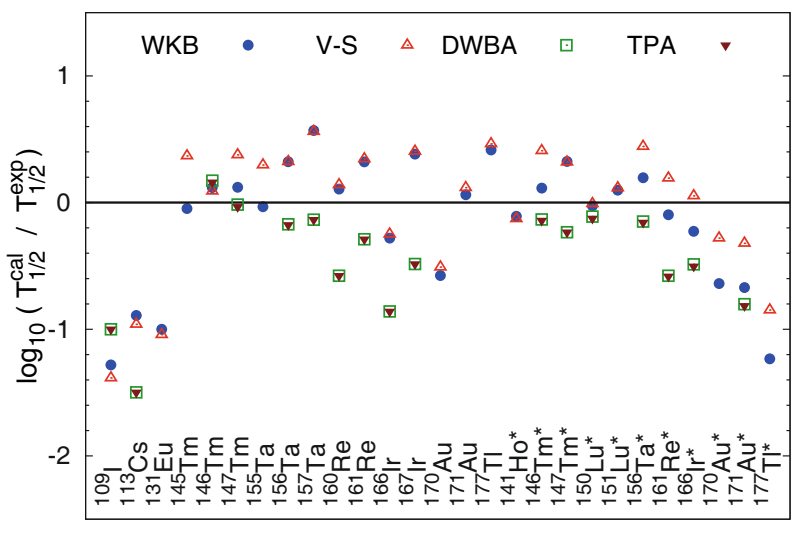

Fig. 4. The deviations between calculated and measured logarithms of proton emission half-lives. Blue circles refer to results obtained using eq. (6), red triangles show the accuracy of the modified Viola-Seaborg formula [17] and green squares and brown triangles refer to DWBA [7] and TPA [8] models, respectively (based on results given in ref. [4]).

are not taken into account. The accuracy in reproducing the experimental data achieved using the present WKB model, Viola-Seaborg formula developed for proton emission [17], DWBA [7] and TPA [8] approximations is shown in fig. 4. Our results obtained within the WKB formalism with only one parameter $r_{0}$ are comparable with those obtained using the four adjustable parameter formula of ref. [17] (r.m.s. = 0.370). For both models the agreement with experiment is slightly worse for the lightest $\left({ }^{109} \mathrm{I}\right.$, $\left.{ }^{177} \mathrm{Cs}\right)$ and the heaviest $\left({ }^{177} \mathrm{Tl}^{*}\right)$ emitters. The accuracy of DWBA and TPA approaches is lower (r.m.s. $\simeq 0.60$ in both cases). The half-lives calculated in these two models underestimate the experimental data in most cases.

There are two experimental quantities which enter into our calculations: the $Q$-value and the angular momentum $l$. It is worthwhile to investigate how the obtained values depend on the precision of these experimental data. The half-lives of ${ }^{109} \mathrm{I},{ }^{131} \mathrm{Eu}$ and ${ }^{177} \mathrm{Tl}^{*}$ are relatively far from the global trend. To explain the source of this large discrepancy we analyzed these isotopes separately. In fig. 5 


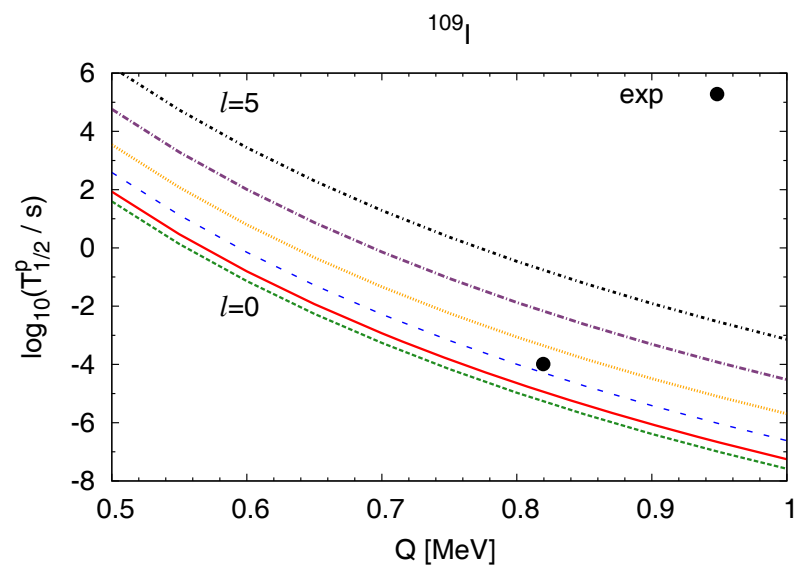

Fig. 5. The $Q$-value dependence of the half-life of proton radioactivity for ${ }^{109} \mathrm{I}$ for various values of angular momenta $\left(l_{\exp }=0[23]\right)$.

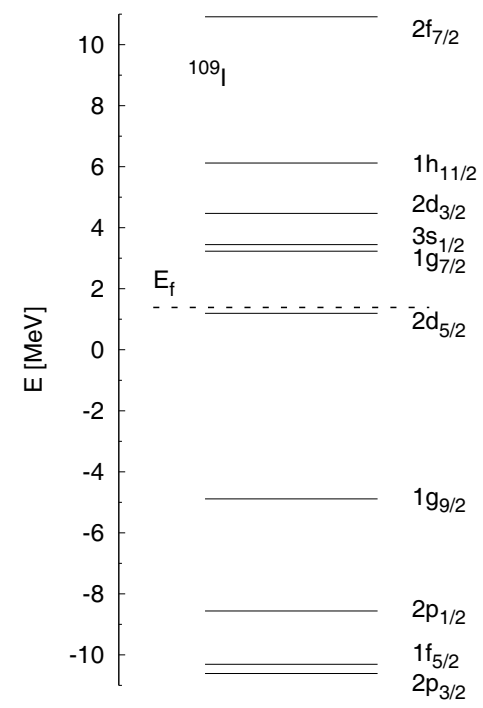

Fig. 6. Sequence of proton levels of ${ }^{109} \mathrm{I}$ in its spherical shape. Labels on the right denote quantum numbers: principal $(n)$, orbital $(l)$ and spin $(j)$. The Fermi level is plotted as the black dashed line.

we present the logarithms of proton emission half-lives calculated for various angular momenta $l$ and $Q$-values. A high sensitivity of the results on $l$ is visible. The change of $l$ by one unit up (down) (for $l \geq 3$ ) gives a half-life longer (shorter) by more than one order of magnitude or more for the same $Q$-value. The dependence of the results on the $Q$-value is not so pronounced. Variation of the order of $0.1 \mathrm{MeV}$ changes the half-life even by 0.6 orders of magnitude. The observed half-life for ${ }^{109} \mathrm{I}$ is reproduced well for the experimental $Q$-value but for $l=2$ which is larger than the experimental $l_{\exp }=0[23]$. To understand these results, we analyzed the single particle energies from microscopic calculations using the HartreeFock-Bogolubow model with the Gogny-type force D1S parameter set $[30,31]$. As one may deduce from fig. 6 , it is possible to observe the proton emitted with angular momentum $l=2$ from the $5 / 2$ state, which is consistent with

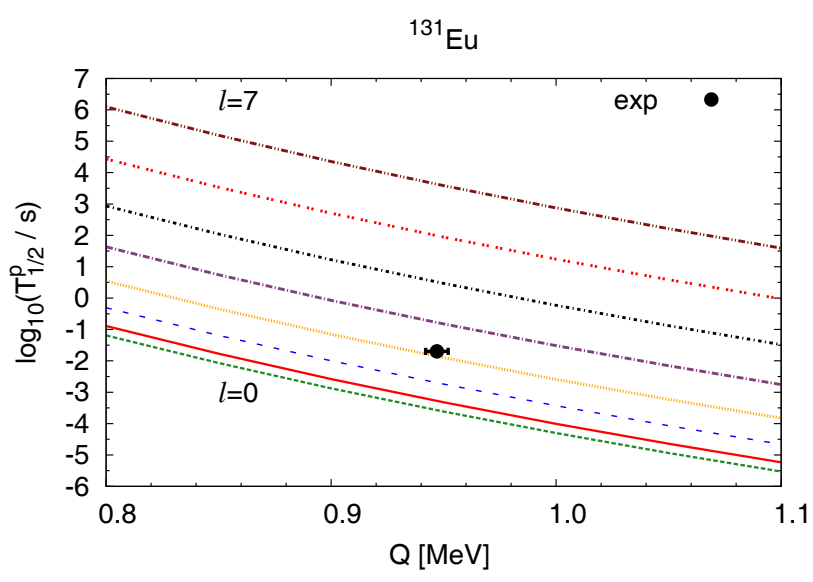

Fig. 7. Similar to fig. 5 but for ${ }^{131} \mathrm{Eu}\left(l_{\exp }=2\right)$.

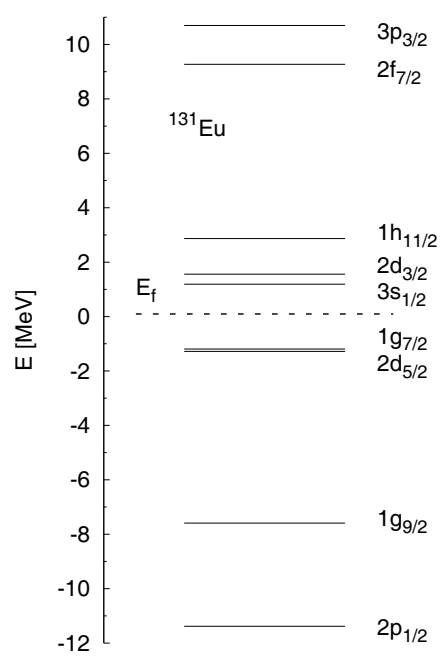

Fig. 8. The same as in fig. 6 but for ${ }^{131} \mathrm{Eu}$.

our predictions. Similarly, for ${ }^{131} \mathrm{Eu}$ the data are reproduced for $l=3$ (fig. 7) rather than for $l_{\exp }=2$ [23]. The level scheme obtained presented in fig. 8 shows that the ground state of ${ }^{131} \mathrm{Eu}$ corresponds to $7 / 2^{+}$, which suggests that the proton is emitted with $l=4$. It is a larger angular momentum than that used to reproduce data in our model as can be seen in fig. 7. Our HFB calculations with the Gogny force predict that ${ }^{131} \mathrm{Eu}$ is well deformed in its ground state $\left(Q_{2}=7 \mathrm{~b}\right)$, which can significantly decrease the proton emission half-life with respect to our estimate obtained in the model made for spherical and nearly spherical nuclei. The results, given in brackets in table 1, suggest that the main reason for the discrepancies is the value of the angular momentum used in the analysis, which is not directly measured in experiment, but deduced from the comparison of the measured proton energies and the spectroscopic factors with theoretical estimates $[3,32]$. A similar discussion to that based on figs. 5 and 7 for ${ }^{109} \mathrm{I}$ and ${ }^{131} \mathrm{Eu}$ isotopes is presented in fig. 9 for the isomer ${ }^{177} \mathrm{Tl}^{*}$ : it is suggested that the proton is emitted with $l=6$ which is improbable when one looks at fig. 10 where the single-particle levels of ${ }^{177} \mathrm{Tl}$ are drawn. 


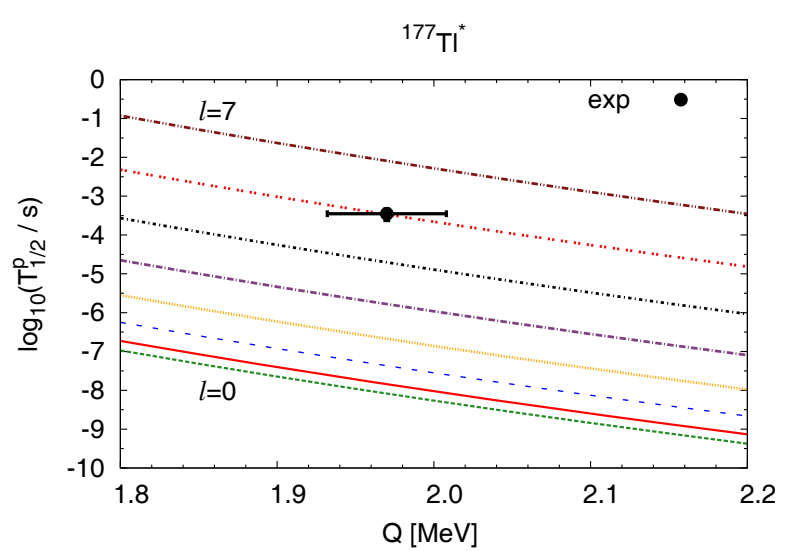

Fig. 9. Similar to fig. 5 but for ${ }^{177} \mathrm{Tl}^{*}\left(l_{\exp }=5\right)$.

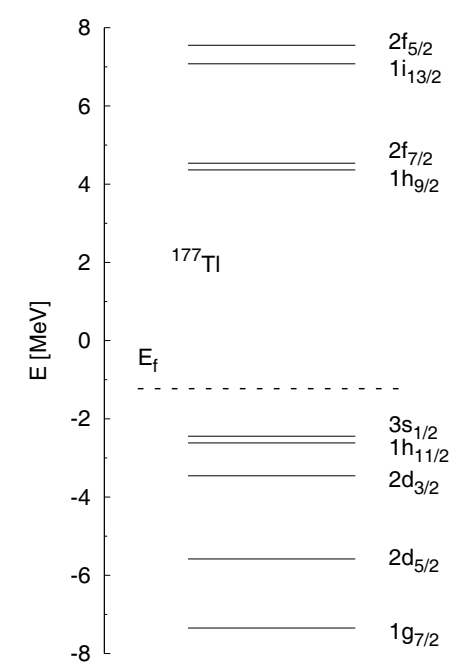

Fig. 10. The same as in fig. 6 but for ${ }^{177} \mathrm{Tl}^{*}$.

The first $l=6$ state $I=13 / 2^{+}$is about $9.5 \mathrm{MeV}$ above the $I=1 / 2^{+}$ground state. Thus the angular momentum $l=6$ cannot be associated with the ${ }^{177} \mathrm{Tl}^{*}$ isomer which has an excitation energy of only $807 \mathrm{keV}$. The level scheme obtained with a Gogny force instead suggests that this isomer corresponds to the hole state $h_{11 / 2}$. The discrepancy between the prediction of our model and the data cannot originate from the deformation effect, as the deformation lowers both Coulomb and centrifugal barriers, which can only decrease the decay half-life with respect to the estimates of our model. Therefore the origin of this large discrepancy of the proton decay half-life of ${ }^{177} \mathrm{Tl}^{*}(1.24$ orders of magnitude) becomes rather unclear and probably has its source in microscopic effects not included in our simple model.

\section{Summary}

The following conclusions can be drawn from our investigations:

- A simple one-parameter model based on Gamow theory of alpha emission is used to calculate proton decay half-lives of nuclei.
- Good accuracy is achieved in the reproduction of the experimental data using only one parameter (radius constant) which has a clear physical meaning.

- We have shown that using the same simple formalism one can describe three decay modes: alpha, cluster and proton emission [19]. One obtains the best fits in all modes for the same $r_{0}=1.21 \mathrm{fm}$.

- Our analysis suggests that the proton is emitted from the $l=2$ state in ${ }^{109} \mathrm{I}$ and from the $l=3$ state in ${ }^{131} \mathrm{Eu}$, which contradicts the predictions made in [23].

- The accuracy of the presented one-parameter model in reproducing the experimental data is comparable with that obtained using the Viola-Seaborg formula of refs. [15-17] which contains four adjustable parameters, fitted to proton decay only, and is better in comparison to more sophisticated theoretical models.

- The presented model may be useful for the evaluation of decay rates of different competing processes, revealing trends in the lifetimes of isotope chains, as well as in conceiving measurements of unknown proton emitters in future experiments.

This work was partially supported by the Polish National Science Centre grant No. 2013/11/B/ST2/04087 and The PolishFrench COPIN IN2P3 collaboration agreement under project number 15-A and 08-131.

Open Access This is an open access article distributed under the terms of the Creative Commons Attribution License (http://creativecommons.org/licenses/by/4.0), which permits unrestricted use, distribution, and reproduction in any medium, provided the original work is properly cited.

\section{References}

1. K.P. Jackson, C.U. Cardinal, H.C. Evans, N.A. Jelley, J. Cerny, Phys. Lett. B 33, 281 (1970).

2. J. Cerny, J.E. Esterl, R.A. Gough, R.G. Sextro, Phys. Lett. B 33, 284 (1970).

3. S. Hofmann, in Particle emission from nuclei, edited by D.N. Poenaru, M. Ivascu, Vol. 2 (CRC, Boca Raton, 1989) Chapt. 2.

4. S. Aberg, P.B. Semmes, W. Nazarewicz, Phys. Rev. C 56, 1762 (1997)

5. G.L. Poli et al., Phys. Rev. C 59, R2979 (1999).

6. S.B. Duarte et al., At. Data Nucl. Data Tables 80, 235 (2002).

7. H. Feshbach, Theoretical Nuclear Physics: Nuclear Reactions (Wiley, New York, 1992).

8. S.A. Gurvitz, Phys. Rev. A 38, 1747 (1988).

9. V.P. Bugrov, S.G. Kadmensky, Phys. At. Nucl. 49, 967 (1989).

10. V.P. Bugrov, S.G. Kadmensky, Phys. At. Nucl. 59, 339 (1996).

11. E. Maglione, L.S. Ferreira, R.J. Liotta, Phys. Rev. Lett. 81, 538 (1998).

12. C. Xu, Z. Ren, Phys. Rev. C 68, 034319 (2003).

13. C. Xu, Z. Ren, Phys. Rev. C 69, 024614 (2004). 
14. D.S. Delion, R.J. Liotta, R. Wyss, Phys. Rev. Lett. 96, 072501 (2006).

15. D. Ni, Z. Ren, T. Dong, C. Xu, Phys. Rev. C 78, 044310 (2008).

16. D. Ni, Z. Ren, Rom. J. Phys. 57, 407 (2012).

17. J.M. Dong, H.F. Zhang, G. Royer, Phys. Rev. C 79, 054330 (2009).

18. B. Buck, A.C. Merchant, S.M. Perez, Phys. Rev. C 45 , 1688 (1992).

19. A. Zdeb, M. Warda, K. Pomorski, Phys. Rev. C 87, 024308 (2013).

20. S.G. Nilsson, Dan. Mat.-Fys. Medd. 29, No. 16 (1955).

21. W.D. Myers, W.J. Swiatecki, Ann. Phys. 55, 395 (1969).

22. W.D. Myers, W.J. Swiatecki, Ann. Phys. 84, 186 (1974).
23. G. Audi, F.G. Kondev, M. Wang, B. Pfeiffer, X. Sun, J. Blachot, M. MacCormick, Chin. Phys. C 36, 1157 (2012).

24. A.P. Robinson et al., Eur. Phys. J. A 25, 155 (2005).

25. I.G. Darby et al., Phys. Rev. C 83, 064320 (2011).

26. R.D. Page et al., Phys. Rev. C 53, 660 (1996).

27. C.N. Davids et al., Phys. Rev. C 55, 2255 (1997).

28. H. Kettunen et al., Phys. Rev. C 69, 054323 (2004).

29. T.N. Ginter et al., Phys. Rev. C 68, 034330 (2003).

30. J.F. Berger, M. Girod, D. Gogny, Nucl. Phys. A 428, 23c (1984).

31. J.F. Berger, M. Girod, D. Gogny, Comput. Phys. Commun. 63, 365 (1991).

32. P.J. Woods, C. Davids, Annu. Rev. Nucl. Part. Sci. 47, 541 (1997). 